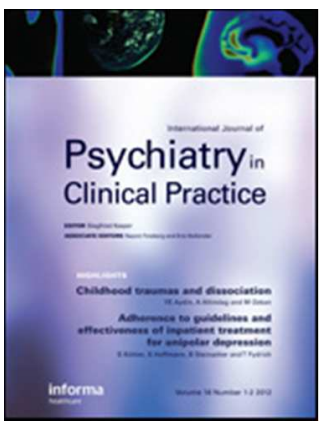

\title{
Repetitive Transcranial Magnetic Stimulation (rTMS) for depression: outcomes in a United Kingdom (UK) clinical practice
}

\begin{tabular}{|r|l|}
\hline Journal: & International Journal of Psychiatry in Clinical Practice \\
\hline Manuscript ID & MPCP-2018-0028.R2 \\
\hline Manuscript Type: & Original Article \\
\hline Date Submitted by the Author: & n/a \\
\hline Complete List of Authors: & $\begin{array}{l}\text { Griffiths, Chris; Northampton Healthcare NHS Trust; University of } \\
\text { Northampton } \\
\text { O'Neill-Kerr, Alex; Northamptonshire Healthcare NHS Foundation Trust } \\
\text { Millward, Tim; Northamptonshire Healthcare NHS Foundation Trust } \\
\text { da Silva, Ksenija; University of Northampton }\end{array}$ \\
\hline Keywords: & $\begin{array}{l}\text { Repetitive Transcranial Magnetic Stimulation, treatment resistant } \\
\text { depression, service delivery, naturalistic }\end{array}$ \\
\hline
\end{tabular}




\section{Title sheet}

Title: Repetitive Transcranial Magnetic Stimulation (rTMS) for depression: outcomes in a United Kingdom (UK) clinical practice

Running heading: rTMS for depression: clinical practice outcomes

Authors: Chris Griffiths 1; Alex O'Neill-Kerr 1; Timothy Millward 1; Ksenija Da Silva 2

1 - Innovation and Research department, Northamptonshire Healthcare NHS Foundation Trust, Northampton, UK

2 - Faculty of Health and Society, University of Northampton, Northampton, UK

Corresponding author: Dr Chris Griffiths, Northamptonshire Healthcare NHS Foundation Trust, Berrywood Hospital, Northampton, NN5 6UD, +44 (0)1604 685528, Chris.Griffiths@northampton.ac.uk

Professor Alex O'Neill-Kerr - Northamptonshire Healthcare NHS Foundation Trust, UK Northamptonshire Healthcare NHS Foundation Trust, Berrywood Hospital, Northampton, NN5 6UD

Email: Alex.Kerr@nhft.nhs.uk

Tel: 01604682623

Dr - Northamptonshire Healthcare NHS Foundation Trust, UK

Northamptonshire Healthcare NHS Foundation Trust, Berrywood Hospital, Northampton, NN5 6UD

Tel: 01604685527

Dr Ksenija Da Silva - University of Northampton, UK

Address: Park Campus, Boughton Green Rd, Northampton NN2 7AL, UK

Email: Ksenija.DaSilva@northampton.ac.uk

$44(0) 1604892344$

Declaration of interests

Professor Alex O'Neill-Kerr is a consultant to Magstim. No other authors have any conflicts of interests to declare. 
Title: Repetitive Transcranial Magnetic Stimulation (rTMS) for depression: outcomes in a United Kingdom (UK) clinical practice

\section{Abstract \\ Objective:}

The aim of this paper is to present the outcomes data from the largest United Kingdom's (UK) National Health Service (NHS) clinical rTMS service treating treatment resistant depression (TRD).

Methods

The study was a retrospective investigation of routinely collected data on patients receiving rTMS between 2015 and 2017. Measures used were the clinician-rated Clinical Global Impression (CGI) and Hamilton Depression Rating Scale (HAM-D), and patient rated Beck Depression Inventory (BDI). The outcome data of 73 patients with TRD was analysed. The sample included patients with co-morbid psychiatric diagnosis.

Results

Response and remission rates respectively were $40.4 \%$ and $25.5 \%$ for the HAM-D; $35.6 \%$ and $20.8 \%$ for the BDI; and $51.1 \%$ and $52.1 \%$ for the CGI. Effect sizes were medium (.54, .52 and .56 respectively).

Conclusions

The results show that a UK based clinical service achieves similar results to those published internationally and that clinical rTMS can have significant impact on symptoms of depression in many patients with TRD. Health services are under pressure to make financial savings, investment in rTMS could reduce the long term treatment costs associated with TRD.

Key words: Repetitive Transcranial Magnetic Stimulation (rTMS), treatment resistant depression, service delivery, naturalistic 
Introduction

Major depressive disorder (clinical depression) is the leading cause of disability worldwide, and is a major contributor to the overall global burden of disease (WHO, 2017). The causes of depression vary at an individual level and can be a result of a complex interaction of social, psychological and biological factors. Depression can result in emotional, psychological and functional problems that can be detrimental to the well-being and health of those affected and their friends and family (WHO, 2017). Depression carries a large cost to society through care and treatment costs and the loss of productivity and societal contribution of those affected (Greenberg et al., 2015). Even where pharmacological and psychological treatment is available, not everyone will find they are effective for them; relapse rates remain significant, underscoring the chronicity of depressive disorders (Huynh \& Mclntyre, 2008)

There is no single accepted definition of what constitutes treatment resistant depression (TRD) (Berlim \& Turecki, 2007). A study in the US reported over $50 \%$ of people did not experience remission after first-line antidepressant treatment, and one-third did not experience remission after four different courses of treatment (Rush et al., 2006). A multi-site study in Europe reported that $50 \%$ of people did not respond to two consecutive courses of antidepressant treatment (Souery et al., 2008). The non-response rate to psychotherapy, most commonly cognitive behavioural therapy (CBT), has been reported as being $70 \%$ (Griffiths \& Griffiths, 2015). Therefore, many people do not respond to 'standard' treatments that are generally available in first world economies, and some who do respond initially may relapse and become unresponsive to subsequent treatment.

A relatively new treatment being offered by state and private healthcare providers is repetitive transcranial magnetic stimulation (rTMS). This is a form of neuromodulation: a non-invasive and non-convulsive technique where a purpose-made electromagnetic coil is placed against the patient's scalp to deliver a short, powerful magnetic field pulse to induce electric currents in the cerebral cortex (Hardy et al., 2016). rTMS treatment usually comprises daily sessions lasting about 30 minutes, over a period which is typically for 4 to 6 weeks (Hardy et al., 2016). Evidence suggests that rTMS results in changes in brain activity, metabolism and connectivity that relate to emotional processing (Kito S, Fujita K, \& Koga, 2008). However, as with many forms of antidepressant treatment the exact mechanism of treatment action is unknown (Hardy et al., 2016).

In the United States the Food and Drug Administration (FDA) approved TMS for treatment of depression in 2008 (Janicak \& Dokucu, 2015). In the UK, the National Institute for Health and Care Excellence (NICE) (IPG 542, 2015) appraised the evidence for rTMS in treatment resistant depression (TRD) and found it to be safe and effective in reducing depressive symptoms compared to sham TMS, and that treatment does not require either hospital admission or anaesthesia (NICE, 2015). Treatment can be carried out on an outpatient basis and rTMS was recommended for the treatment of depression, including TRD. NICE (IPG $542,2015)$ noted that reports from patients about the treatment were positive, and patients described significant benefits to their quality of life, including some no longer taking oral antidepressant medications (NICE, 2015). A systematic review of 45 RCTs found rTMS to be robustly effective versus sham TMS on depression symptoms, response or remission; and that patients undergoing rTMS are twice as likely to achieve clinical response or remission 
compared to a sham procedure (Health Quality Ontario, 2016). Yesavage et al (2018) found that $39.0 \%$ of war veterans in an rTMS RCT experienced remission, but there was no difference in remission rates between the active and sham treatments. However, this trial included participants with comorbid PTSD and/or substance abuse and the population was largely male (and so not representative of general TRD population); as Nemeroff (2018) states, placebo rates can be a major issue in depression research. A recent review found that placebo response in rTMS depression trials was large, suggesting that excluding placebo responders with a run-in phase may not confer advantage, since response to rTMS may decrease as well (Razza et al. 2018). Placebo response may be a component of therapeutic response to rTMS, and placebo response increase over time could indicate need for improvement in rTMS trial designs, including better sham versions of rTMS (Razza et al. 2018).

In research trials, response and remission rates have ranged between $25-50 \%$ and $12-35 \%$ respectively. (Allan, Herrmann, \& Ebmeier, 2011; Berlim et al. 2014; Gross, Nakamura, Pascual-Leone, \& Fregni, 2007; Herrmann \& Ebmeier, 2006; Kozel \& George, 2002; Lam, Chan, Wilkins-Ho, \& Yatham, 2008; Schutter, 2010; Slotema, Dirk Blom, Hoek, \& Sommer, 2010; Xie, Chen, \& Wei, 2013). Following initial FDA clearance in 2008, a number of peer review published studies have reported remission and therapeutic response in clinical service settings. Response and remission rates have ranged between $29-51 \%$ and $6-37 \%$ respectively (Carpenter et al, 2012; Connolly et al. 2012; Galletly, Clarke, Carnell, \& Gill, 2015; Taylor et al., 2017).

There is considerable research evidence for the effectiveness of repetitive transcranial magnetic stimulation in the treatment of depression from research trials. However, there has been a lack of information on outcomes from UK clinical practice. This is the first study to report the patient characteristics and outcomes data from a service delivering rTMS within the United Kingdom's National Health Service (NHS).

\section{Methods}

$\underline{\text { Design }}$

The study was a retrospective investigation of routinely collected data on patients receiving rTMS services between 2015 and 2017 at a UK based service provider. Demographic information (gender, age at admission), diagnosis, treatment funder, and outcomes data were extracted from clinical records containing routinely collected data. Analysis was conducted using an anonymised database or routinely collected data and so ethical approval was not required.

\section{$\underline{\text { Measures }}$}

The Hamilton Depression Rating Scale (HAM-D) is one of the most commonly used and extensively studied measures of depressive symptoms (Hamilton, 1960). Internal, inter-rater and retest reliability estimates are adequate for the global score (Hamilton, 1960; Bagby, Ryder, Schuller, \& Marshall, 2004). The 2 item version was used, first 17 items scored. The Beck Depression Inventory (BDI) is one of the most widely used measures of depression severity (Beck \& Alford, 2009). The scale has high content validity, construct validity, 
concurrent validities, content validity internal consistency, and reliability (Jackson-Koku, 2016). The Clinical Global Impression score (CGI) rating scale is one of the most widely used assessment instruments in psychiatry (Guy, 1976). The Clinical Global Impression Scale (CGI) is a brief clinician-rated instrument of illness severity. There is a lack of strong evidence for the validity and therefore it is recommended as part package of assessments (Forkmann et al. 2011). The measures were collected prior to treatment and shortly following final first course of treatment.

\section{Process to treatment}

Patients with depressive symptoms are referred to the service by their GP or psychiatrist. A medical and physical history is taken and patients are then assessed for a diagnosis of TRD by a psychiatrist working in the neuromodulation unit. TRD is defined as non-response to 2 or more appropriate courses of anti-depressants. rTMS protocol and length of treatment are set by the psychiatrist. Patients who lack capacity to consent to treatment are excluded. Patients are provided with information about the treatment (procedures, risks, side effects, remission/response rates) and are required to sign a 'consent to treatment' form; they can withdraw consent at any point. rTMS equipment supplier is Magstim.

\section{$\underline{\text { rTMS treatment }}$}

The site of stimulation is be determined using the min- $\mathrm{R}$ "10-20" method with stimulation at the F5 electrode (Tsuzuki et al. 2016). The majority of patients ( $n=68,93.2 \%)$ received of FDA (Food and Drug Administration, 2011) depression protocol high frequency stimulation to left dorsolateral prefrontal cortex. Five (6.8\%) received Hadley et al. (2011) depression protocol high frequency stimulation to left dorsolateral prefrontal cortex. Of these, 18 $(24.7 \%)$ patients with depression and generalised anxiety (as measured with the GAD-7 [Spitzer et al. 2006]) additionally received right DLPFC inhibitory rTMS (Dilkov et al. 2017), immediately prior to delivery of FDA left dorsolateral prefrontal cortex depression treatment. This additional treatment option was added to the service in October 2016. The average strength of magnetic field was $63.84 \%(S D=6.224$, range: $43-75)$. During this period there were no seizures and two syncopal episodes. Analysis

Analysis of change from baseline to post first course treatment scores was carried out using data from 73 patients. Not all patients had data sets for all depression measures and so numbers per measure vary. For categorical responses, we defined treatment response as a $50 \%$ or greater drop on the last assessment of treatment. Partial response was defined as a $25-50 \%$ drop on the last assessment. Remission was defined as CGI-S score as $\leq 2$, BDI $\leq$ 9 and HAM-D $\leq 7$ (Health Quality Ontario, 2016; Connolly et al. 2012; Galletly, Clarke, Carnell, \& Gill, 2015).

As the continuous variables were not normally distributed, Wilcoxon signed-rank tests $(Z)$ were used to compare baseline with post-treatment measures, together with the calculated effect sizes. Using non-parametric analysis (Pearson chi square test and Mann-Whitney $U$ test), the differences in demographic variables and between responders and non-responders on a number of variables were explored. All tests were 2-sided, at $1 \%$ level of statistical significance. Spearman's rho was used to calculate correlations. Data were analysed using statistics software package SPSS. 


\section{Results \\ Patient characteristics}

The data was collected on a sample of 73 patients with TRD, who were treated between January 2015 and October 2017, see table 1. Cross tabulation indicated that female patients were overrepresented $[X 2(1)=6.041, p=.014]$. There were no differences between HAM-D responders and non-responders in age $(N=73, U=106.0, p=.175)$ or gender $(N=73, X 2=$ $0.147, p=.701)$. Similarly, there were no differences in age and gender between responders and non-responders as measured by the $\mathrm{CGI}$ and BDI scores.

Table 1. Demographic characteristics of the sample $(n=73)$.

Co-morbid diagnosis was as follows: generalized anxiety disorder (GAD) ( $n=33,42.5 \%)$; bipolar affective disorder $(n=10,13.7 \%)$; psychosis $(n=4,5.5 \%)$; emotionally unstable personality disorder, autism spectrum disorder (ASD), chronic fatigue syndrome, bulimia nervosa $(n=3,4.1 \%)$; post-traumatic stress disorder (PTSD), alcohol dependence $(n=2$, $2.8 \%$ ); chronic pain syndrome, cocaine dependence, schizoaffective disorder, dysthymia, fibromyalgia, mixed and other personality disorder, narcissistic personality disorder, obsessive-compulsive disorder, Parkinson's disease, social phobia generalised, intentional self-harm $(n=1,1.4 \%)$.

\section{$\underline{\text { rTMS Treatment Outcome }}$}

The average number of rTMS sessions across acute phase was 26.68 (SD $=9.451$, range: 10-60). An average baseline depression scores was in the moderate-severe range at the baseline (Table 2). There was a statistically significant improvement on all depression measures after the rTMS treatment, with medium effect sizes.

Table 2. Mean pre- and post-treatment scores and mean change in scores

\section{Categorical Response and Remission Rates}

The highest response and remission rates were elicited by the CGI, then HAM-D and finally BDI (see Table 3). However, the highest partial response was elicited by the HAM-D, then CGI and finally BDI. There was a statistically significant positive correlation $(p<.001)$ between all three measures: 356 between HAM-D and BDI; .369 between HAM-D and CGI; and .519 between $\mathrm{CGI}$ and $\mathrm{BDI}$. Using binary logistic regression to explore whether age or sex was a predictor of response and remission rates yielded no statistically significant predictors of the primary outcome.

Table 3. Response, partial response and remission rates (\%) 


\section{Discussion}

The results show that rTMS delivered in a UK based clinical service is effective. The outcomes are comparable to published RCTs and service data. (Carpenter et al, 2012; Connolly et al. 2012; Galletly, Clarke, Carnell, \& Gill, 2015; Health Quality Ontario, 2016; Taylor et al., 2017) This indicates the effectiveness of NHS delivered rTMS and the need to increase availability to enable more people with TRD to potentially benefit.

In comparing our results with other published service data, the HAM-D remission rates at $25.5 \%$ were higher than one study (20\%) but lower than two others $(28 \%$ and $35.3 \%)$; however, the response rates at $40.4 \%$ were very similar or better than three published studies (Carpenter et al, 2012; Connolly et al. 2012; Galletly, Clarke, Carnell, \& Gill, 2015). In terms of the BDI results, remission and response rates at $20.8 \%$ and $35.6 \%$ respectively were almost identical to published service data (Connolly et al. 2012). The CGI remission rates of $52 \%$ and response rates of $51.1 \%$ were better than published data (Connolly et al. 2012; Taylor et al., 2017).

It is not possible to say with certainty why these differences exist, the contribution of differences in patient or service factors could be the subject of further investigation. The differing length of the treatment could be a factor; there is no gold standard as to how long the treatment should last, clinical judgment (usually made in consultation with the patient) is used to determine when to end treatment.

There can be a number of reasons for the differences in response and remission rates seen between the measures. For example, correlations between the measures were found to be moderate; different scales may pick up different factors in depression; and have differing sensitivity to the effects of the treatment may contribute to the varying degrees of response and remission rates. One of measures was patient completed (BDI) and two clinician completed (CGI \& HAM-D), and the assessment of the level of depression symptoms by the patient can differ to that of the clinician.

Participants in clinical trials tend to be narrowly defined in terms of diagnosis. The UK based service which is the basis of this paper assesses people as having TRD without excluding those with co-morbid psychiatric diagnosis. The outcome results indicate that this approach is effective in targeting depression irrespective of the existence of co-morbid mental illness. This offers hope to the many people with complex mental health histories who experience clinical depression that has not responded to other treatments.

rTMS could reduce long term health and social care costs associated with TRD. Clinical depression incurs $£ 1.7$ billion per year UK service costs (2007 figures) and this is estimated to rise to $£ 2$ billion (2007 prices) in 2026 (McCrone et al. 2008) Around 75\% of these costs are attributable to treatment resistant depression (TRD) (Mrazek, Hornberger, \& Altar, Degtiar, 2014). People with persistent depression seen in a specialist depression service offering NICE recommended psychotherapy and pharmacotherapy incur costs of $£ 7-8,000 \mathrm{a}$ year (Morriss et al. 2016). Between 12\%-20\% of depressed patients have TRD, and having TRD results in greater loss of economic contribution (Eaton et al. 2008; Nemeroff, 2007). Cost-benefit analysis is required to understand the potential savings that could be derived by the wider implementation of rTMS services for people with TRD. 
Further research is required as to why some people respond and others do not and what factors determine response. This research is vital due to the financial cost and the treatment delivery time involved in undergoing a course of rTMS treatment.

Limitations

Data was extracted from a clinical database and patient notes with some missing assessments, evidenced by the different number of subjects available for analysis on each outcome measure. Treatment was open label and adjunct to any existing antidepressant treatments, with the absence of a control. Data was from single site in the UK limiting generalizability, however; patients were from across the UK, partially negating this. Females were overrepresented and so the results are less generalizable to males.

Conclusions

This study adds to the findings of other published service data that outpatient delivered rTMS clinical is effective. Further work is needed to define the role of rTMS in a depression pathway. This work needs to understand when it is best to offer rTMS in people's experience of depression and when rTMS is a better option than other treatment options such as switching antidepressants or ECT. The authors view is that due to low side effects and lack of a need for a general anaesthetic that it should be considered in advance of using ECT.

The availability of rTMS is currently limited. The results support wider availability of rTMS as a treatment option for people with TRD. Ideally, rTMS should be a treatment option which is freely available to people with TRD who meet the criteria for treatment rather than just those who can afford the costs of private treatment. Training in rTMS delivery and advice on service set up of rTMS services is essential in expanding availability.

\section{Key points}

- Clinical practice delivered rTMS can have significant impact on symptoms of depression in patients with TRD

- $\quad$ rTMS is effective in treating depression irrespective of the existence of co-morbid mental illness

- rTMS should be a treatment option which is freely available to people with TRD who meet the criteria for treatment

\section{Acknowledgements}

None

\section{Declaration of interests}

Professor Alex O'Neill-Kerr is a consultant for Magstim. The other authors have no conflicts of interest, and the work was not supported or funded by a company. 


\section{References}

Allan, C. L., Herrmann, L. L., \& Ebmeier, K. P. (2011). Transcranial magnetic stimulation in the management of mood disorders. Neuropsychobiology, 64(3), 163-169.

Bagby, R. M., Ryder, A. G., Schuller, D. R., \& Marshall, M. B. (2004). The Hamilton Depression Rating Scale: has the gold standard become a lead weight?. American Journal of Psychiatry, 161(12), 2163-2177.

Beck, A. T. (1972). Depression: Causes and Treatment. Philadelphia: University of Pennsylvania Press.

Berlim, M. T., \& Turecki, G. (2007). What is the meaning of treatment resistant/refractory major depression (TRD)? A systematic review of current randomized trials. European Neuropsychopharmacology, 17(11), 696-707.

Berlim, M. T., Van den Eynde, F., Tovar-Perdomo, S., \& Daskalakis, Z. J. (2014). Response, remission and drop-out rates following high-frequency repetitive transcranial magnetic stimulation (rTMS) for treating major depression: a systematic review and meta-analysis of randomized, double-blind and sham-controlled trials. Psychological medicine, 44(2), 225239.

Carpenter, L. L., Janicak, P. G., Aaronson, S. T., Boyadjis, T., Brock, D. G., Cook, I. A., ... \& Demitrack, M. A. (2012). Transcranial magnetic stimulation (TMS) for major depression: a multisite, naturalistic, observational study of acute treatment outcomes in clinical practice. Depression and anxiety, 29(7), 587-596.

Connolly, K. R., Helmer, A., Cristancho, M. A., Cristancho, P., \& O'Reardon, J. P. (2012). Effectiveness of transcranial magnetic stimulation in clinical practice post-FDA approval in the United States: results observed with the first 100 consecutive cases of depression at an academic medical center. The Journal of clinical psychiatry, 73(4), e567-73.

Dilkov, D., Hawken, E. R., Kaludiev, E., \& Milev, R. (2017). Repetitive transcranial magnetic stimulation of the right dorsal lateral prefrontal cortex in the treatment of generalized anxiety disorder: A randomized, double-blind sham controlled clinical trial. Progress in NeuroPsychopharmacology and Biological Psychiatry, 1(78), 61-65.

Eaton, W. W., Martins, S. S., Nestadt, G., Bienvenu, O. J., Clarke, D., \& Alexandre, P. (2008). The burden of mental disorders. Epidemiologic reviews, 30(1), 1-14.

Food and Drug Administration (2011). Class II Special Controls Guidance Document: Repetitive Transcranial Magnetic Stimulation (rTMS) Systems - Guidance for Industry and FDA Staff. U.S. Department of Health and Human Service: Maryland.

Forkmann, T., Scherer, A., Boecker, M., Pawelzik, M., Jostes, R., \& Gauggel, S. (2011). The clinical global impression scale and the influence of patient or staff perspective on outcome. BMC psychiatry, 11(1), 83.

Galletly, C. A., Clarke, P., Carnell, B. L., \& Gill, S. (2015). A clinical repetitive transcranial magnetic stimulation service in Australia: 6 years on. Australian \& New Zealand Journal of Psychiatry, 49(11), 1040-1047. 
Greenberg, P. E., Fournier, A. A., Sisitsky, T., Pike, C. T., \& Kessler, R. C. (2015). The economic burden of adults with major depressive disorder in the United States (2005 and 2010). The Journal of clinical psychiatry, 76(2), 155-162.

Griffiths, C. A. \& Griffiths, L. J. (2014). Recovery and reliable change rates for patients scoring severe on depression, anxiety or impaired functioning in a psychological therapies service: IAPT. Mental Health Review Journal, 20(1).

Gross, M., Nakamura, L., Pascual-Leone, A., \& Fregni, F. (2007). Has repetitive transcranial magnetic stimulation (rTMS) treatment for depression improved? A systematic review and meta-analysis comparing the recent vs. the earlier rTMS studies. Acta Psychiatrica Scandinavica, 116(3), 165-173.

Guy, W. (1976). ECDEU assessment manual for psychopharmacology. US Department of Health, and Welfare, 534-537.

Hadley, D., Anderson, B. S., Borckardt, J. J., Arana, A., Li, X., Nahas, Z., \& George, M. S. (2011). Safety, tolerability, and effectiveness of high doses of adjunctive daily left prefrontal repetitive transcranial magnetic stimulation for treatment-resistant depression in a clinical setting. The journal of ECT, 27(1), 18-25.

Hamilton, M. (1960). A rating scale for depression. Journal of neurology, neurosurgery, and psychiatry, 23(1), 56.Hardy, S., Bastick, L., O’Neill-Kerr, A., Sabesan, P., Lankappa, S., \& Palaniyappan, L. (2016). Transcranial magnetic stimulation in clinical practice. BJPsych Advances, 22(6), 373-379.

Health Quality Ontario. (2016). Repetitive transcranial magnetic stimulation for treatmentresistant depression: A systematic review and meta-analysis of randomized controlled trials. Ontario health technology assessment series, 16(5), 1.

Herrmann, L. L., \& Ebmeier, K. P. (2006). Factors modifying the efficacy of transcranial magnetic stimulation in the treatment of depression: a review. The Journal of clinical psychiatry, 67(12), 1870-1876.

Huynh, N. N., \& Mclntyre, R. S. (2008). What are the implications of the STAR* D trial for primary care? A review and synthesis. Primary care companion to the Journal of clinical psychiatry, 10(2), 91.

Jackson-Koku, G. (2016). Beck Depression Inventory. Occupational Medicine, 66(2), 174175.

Janicak, P. G., \& Dokucu, M. E. (2015). Transcranial magnetic stimulation for the treatment of major depression. Neuropsychiatric disease and treatment, 11, 1549.

Kito, S., Fujita, K., \& Koga, Y. (2008). Regional cerebral blood flow changes after lowfrequency transcranial magnetic stimulation of the right dorsolateral prefrontal cortex in treatment-resistant depression. Neuropsychobiology, 58(1), 29-36.

Kozel, F. A., \& George, M. S. (2002). Meta-analysis of left prefrontal repetitive transcranial magnetic stimulation (rTMS) to treat depression. Journal of Psychiatric Practice ${ }^{8}, 8(5), 270-$ 275. 
Lam, R. W., Chan, P., Wilkins-Ho, M., \& Yatham, L. N. (2008). Repetitive transcranial magnetic stimulation for treatment-resistant depression: a systematic review and metaanalysis. The Canadian Journal of Psychiatry, 53(9), 621-631.

McCrone, P. R., Dhanasiri, S., Patel, A., Knapp, M., \& Lawton-Smith, S. (2008). Paying the price: the cost of mental health care in England to 2026. King's Fund: London.

Morriss, R., Garland, A., Nixon, N., Guo, B., James, M., Kaylor-Hughes, C., ... \& Dalgleish, T. (2016). Efficacy and cost-effectiveness of a specialist depression service versus usual specialist mental health care to manage persistent depression: a randomised controlled trial. The Lancet Psychiatry, 3(9), 821-831.

Mrazek, D. A., Hornberger, J. C., Altar, C. A., \& Degtiar, I. (2014). A review of the clinical, economic, and societal burden of treatment-resistant depression: 1996-2013. Psychiatric services, 65(8), 977-987.

Nemeroff, C. B. (2007). Prevalence and management of treatment-resistant depression. Journal of Clinical Psychiatry, 68(8), 17.

Nemeroff, C. B. (2018). The Search for Treatments for Veterans With Major DepressionOf Paramount Importance, yet Still Elusive. JAMA Psychiatry. Published online June 27.

NICE (2015). IPG542. Repetitive transcranial magnetic stimulation for depression. Retrieved from https://www.nice.org.uk/guidance/ipg542

Razza, L. B., Moffa, A. H., Moreno, M. L., Carvalho, A. F., Padberg, F., Fregni, F., \& Brunoni, A. R. (2018). A systematic review and meta-analysis on placebo response to repetitive transcranial magnetic stimulation for depression trials. Progress in NeuroPsychopharmacology and Biological Psychiatry, 81, 105-113.

Rush, A. J., Trivedi, M. H., Wisniewski, S. R., Nierenberg, A. A., Stewart, J. W., Warden, D., ... \& McGrath, P. J. (2006). Acute and longer-term outcomes in depressed outpatients requiring one or several treatment steps: a STAR* D report. American Journal of Psychiatry, 163(11), 1905-1917.

Schutter, D. J. L. G. (2010). Quantitative review of the efficacy of slow-frequency magnetic brain stimulation in major depressive disorder. Psychological medicine, 40(11), 1789-1795.

Slotema, C. W., Dirk Blom, J., Hoek, H. W., \& Sommer, I. E. (2010). Should we expand the toolbox of psychiatric treatment methods to include Repetitive Transcranial Magnetic Stimulation (rTMS)? A meta-analysis of the efficacy of rTMS in psychiatric disorders. Journal of Clinical Psychiatry, 71(7), 873.

Souery, D., Oswald, P., Massat, I., Bailer, U., Bollen, J., Demyttenaere, K., ... \& Zohar, J. (2007). Clinical factors associated with treatment resistance in major depressive disorder: results from a European multicenter study. Journal of Clinical Psychiatry, 68(7), 1062-1070.

Spitzer, R. L., Kroenke, K., Williams, J. B., \& Löwe, B. (2006). A brief measure for assessing generalized anxiety disorder: the GAD-7. Archives of internal medicine, 166(10), 1092-1097. 
Taylor, S. F., Bhati, M. T., Dubin, M. J., Hawkins, J. M., Lisanby, S. H., Morales, O., ... \& Watcharotone, K. (2017). A naturalistic, multi-site study of repetitive transcranial magnetic stimulation therapy for depression. Journal of affective disorders, 208, 284-290.

Tsuzuki, D., Watanabe, H., Dan, I., \& Taga, G. (2016). MinR 10/20 system: Quantitative and reproducible cranial landmark setting method for MRI based on minimum initial reference points. Journal of neuroscience methods, 264, 86-93.

WHO (2017). Depression fact sheet. Retrieved from http://www.who.int/mediacentre/factsheets/fs369/en/

Xie, J., Chen, J., \& Wei, Q. (2013). Repetitive transcranial magnetic stimulation versus electroconvulsive therapy for major depression: a meta-analysis of stimulus parameter effects. Neurological research, 35(10), 1084-1091.

Yesavage, J. A., Fairchild, J. K., Mi, Z., Biswas, K., Davis-Karim, A., Phibbs, C. S., ... \& O'Hara, R. (2018). Effect of repetitive transcranial magnetic stimulation on treatmentresistant major depression in US veterans: a randomized clinical trial. JAMA psychiatry.

Table 1. Demographic characteristics of the sample $(n=73)$.

\begin{tabular}{ll}
\hline Characteristic & \\
\hline Age, Mean \pm SD (Min-Max) & $49.10 \pm 16.01(19-80)$ \\
Sex, $\mathrm{n}(\%)$ & $26(35.6)$ \\
$\quad$ Male & $47(64.4)$ \\
$\quad$ Female & $59(80.8)$ \\
Funding, n (\%) & $14(19.2)$ \\
$\quad$ NHS & \\
$\quad$ Private & \\
Treatment, n, Mean \pm SD (Min- & \\
Max) & $65,24.12 \pm 6.69(10-40)$ \\
$\quad$ Depression & $14,12.36 \pm 8.47(4-30)$ \\
$\quad$ Anxiety & \\
Strength of magnetic field (\%), & \\
Mean \pm SD (Min-Max) & $63.84 \pm 6.22(43-75)$ \\
$\quad$ Depression & $61.25 \pm 2.26(60-65)$ \\
$\quad$ Anxiety & \\
Protocol used, n (\%) & $68(93.2)$ \\
$\quad$ Standard FDA & $5(6.8)$ \\
$\quad$ Hadley 2011 & \\
\end{tabular}

Table 2. Mean pre- and post-treatment scores and mean change in scores

\begin{tabular}{llllll}
\hline $\begin{array}{l}\text { Rating } \\
\text { scale }\end{array}$ & $\boldsymbol{N}$ & Mean \pm SD [range] & $\mathbf{Z}$ & $\boldsymbol{p}$ & $\boldsymbol{r}$ \\
\hline $\begin{array}{c}\text { HAM-D } \\
\text { Pre }\end{array}$ & 61 & $21.59 \pm 4.99[9-33]$ & -5.86 & $<.000^{*}$ & 0.54 \\
$\quad$ Post & 55 & $11.42 \pm 6.52[0-24]$ & & & \\
CGI & & & & & \\
$\quad$ Pre & 57 & $4.86 \pm 0.92[3-7]$ & -5.71 & $<.000^{*}$ & 0.56 \\
$\quad$ Post & 48 & $2.58 \pm 1.16[0-5]$ & & & \\
BDI & & & & & \\
\hline
\end{tabular}


Table 3. Response, partial response and remission rates (\%)

\begin{tabular}{lcccc}
\hline & Response & Partial response & No response & Remission \\
\hline HAM-D & 40.4 & 32.7 & 26.9 & 25.5 \\
CGI & 51.1 & 27.7 & 21.3 & 52.1 \\
BDI & 35.6 & 24.4 & 40.0 & 20.8 \\
\hline
\end{tabular}

